\title{
Optical path difference evaluation of laser-soldered optical components
}

\section{T. Burkhardt}

thomas.burkhardt@iof.fraunhofer.de

\section{Hornaff \\ D. Burkhardt}

\section{E. Beckert}

\author{
Fraunhofer Institute for Applied Optics and Precision Engineering IOF, Albert-Einstein-Str. 7, 07745 \\ Jena, Germany \\ Fraunhofer Institute for Applied Optics and Precision Engineering IOF, Albert-Einstein-Str. 7, 07745 \\ Jena, Germany \\ Fraunhofer Institute for Applied Optics and Precision Engineering IOF, Albert-Einstein-Str. 7, 07745 \\ Jena, Germany \\ Numerik Jena GmbH, Im Semmicht 4, 07751 Jena, Germany \\ Fraunhofer Institute for Applied Optics and Precision Engineering IOF, Albert-Einstein-Str. 7, 07745 \\ Jena, Germany
}

We present Solderjet Bumping, a laser-based soldering process, as an all inorganic joining technique for optical materials and mechanical support structures. The adhesive-free bonding process enables the low-stress assembly of fragile and sensitive components for advanced optical systems. Our process addresses high demanding applications, e.g. under high energetic radiation (short wavelengths of $280 \mathrm{~nm}$ and below and/or high intensities), for vacuum operation, and for harsh environmental conditions. Laser-based soldering allows the low stress assembly of aligned sub-cells as key components for high quality optical systems. The evaluation of the optical path difference in fused silica and the radiation resistant $\mathrm{LAK}_{9} \mathrm{G}_{15}$ glass components after soldering and environmental testing shows the potential of the technique. [DOI: http://dx.doi.org/10.2971/jeos.2015.15059]

Keywords: Laser-based soldering, Solderjet Bumping, inorganic joining, lens mount

\section{INTRODUCTION}

Advanced optical systems in numerous fields of application require the mounting and joining of elements with highest accuracies. The effects of the mounting of components, e.g. lenses in mounts, on deformation of optical surfaces, stress induced birefringence, and positional stability are important to consider, especially for systems operating at short wavelengths and high performance applications. These systems and respective sub-assemblies and sub-cells need to maintain their accuracy and stability under environmental influences, such as humidity, temperature cycles, and mechanical loads. High-end microscopy, like multi-wavelength scanning confocal microscopy or wafer inspection and metrology at deep-ultraviolet (DUV) wavelengths, demand micron and sub-micron positional accuracy [1,2]. Applications for high energetic or ionizing radiation (short wavelengths and/or high intensities), for vacuum operation, for harsh environmental conditions, and challenging mechanical loads require the replacement of the organic adhesive or optical cement by a more robust bonding agent. Spaceborne instruments experience such conditions and should exhibit a low outgassing under vacuum operation as signified by a low Total Mass Loss (TML) and Collected Volatile Condensable (CVC) [3]. Such scientific instrumentation benefits from inorganic joining to avoid adhesives and optical cements.

A few techniques overcome bonding with organic adhesives. While mechanical retainers, e.g. flexures, snap rings, or threaded retainers, are a very common and state-of-the-art approach, great effort has to be dedicated to achieve high accuracy, low surface deformations, and low induced stress. A clamp mount technology for high-NA DUV systems with mechanical clamping but requiring highly accurate manufacturing is shown in [4]. Using low melting solder alloys, an UV-resistant jointing technique for lenses and mounts is described in [5]. It relies on form fit bonding (positively locking) for the fastening of an optical element as the optical material is not solderable. Another approach documents the galvanoplastically manufacturing of spring hinge beams and parts of an optical mounting [6]. By direct deposition of the mount to the lens a high positional accuracy is reported. Applications that rely on the transparent bonding of optical components, i.e. within the clear aperture of the system, need to address the degradation of the adhesive itself, e.g. by development of durable fluoride-based optical cements [7], or utilize an interlayer free joining technique. Direct bonding allows the joining of inorganic crystalline and non-crystalline materials without any auxiliary agents [8]. The assembly of high-peak power optical systems by silicate bonding has also been demonstrated [9]. Laser micro-welding with ultrashort laser pulses at high repetition rates focused at the interface of two optically contacted components has been shown for the bonding of glass [10, 11]. The bonding of optical fibers by laser splicing $[12,13]$ is also state-of-the-art but mainly limited to fibers and fiber components [14]. 
We present a fully inorganic joining technique using a laserbased soldering process for the bonding of optical materials and mechanical support structures. The evaluation of the optical path difference (OPD) in fused silica (SQ1) and LAK9G15, a radiation resistant glass, components after soldering and environmental testing shows the suitability of this process for the bonding of sensitive optical elements. We demonstrate Solderjet Bumping for the adhesive-free and low-stress assembly of optical materials, paving the way to aligned sub-cells as key components for high quality optical systems.

\section{METHODOLOGY}

\subsection{Laser-based Solderjet Bumping}

Joining, packaging, and assembly of sensitive optical components benefit from flux-free bonding techniques that prevent contamination of surfaces. Processes that limit heating to a localized zone are also advantageous for high-precision joining. Precisely controlled laser-based heating and solder reflow lead to minimized areas of thermal influence. Localized heating also enables a sequential build-up of systems as thermal dealignment during assembly is avoided. Contact-free heating could be provided by Joule heating using integrated resistive layers as demonstrated by the Pick\&Align soldering technique [15]. Laser irradiation is a very versatile tool to reflow localized solder volumes as shown by the TRIMO approach for surface-mounted optics [16], by thin film laser soldering processes [17], and by Solderjet Bumping [18].

Solderjet Bumping is a laser-assisted soldering process. It uses spherical solder preforms (diameter $40 \mu \mathrm{m}$ to $760 \mu \mathrm{m}$ ) to provide precisely defined solder volumes. Solder spheres are transferred from a reservoir to a placement capillary where they are heated and molten by an IR laser pulse. Nitrogen pressure ejects the liquid solder droplets from this capillary. The solder can be selected according to application needs from a wide range of alloys, e.g. tin-based lead-free solders or high melting eutectic gold-tin. The Solderjet bond head integrates all process steps - separation and feeding of solder preforms, reflow using a laser pulse, and application of liquid solder droplets - in a single and flexible to use machine. Jetting of droplets allows the application to complicated 3D geometries and the liquid droplets optimize thermal contact to the wetting surface of the components to be joined. Compared to thin film soldering techniques bonding of materials with lower surface quality is feasible, since small gaps can be bridged by the solder. Using this advantage it is also possible to adjust components prior to joining in six degrees of freedom. Solderjet Bumping allows the bonding of a broad range of materials in heterogeneous combinations. Components are metallized using thin film techniques such as physical vapor deposition (PVD) or thick film processes, e.g. printed metal pastes. Nobel finish layers (gold flash) provide excellent solder wetting in the flux-free soldering process. We use an established PVD metallization system feasible for different substrate materials that consists of titanium, platinum, and gold. Using Solderjet Bumping we demonstrated sub-micron accuracy packaging of numerous micro-optical assemblies [18], the bonding of polarization maintaining fibers [19], the assembly of a compact and

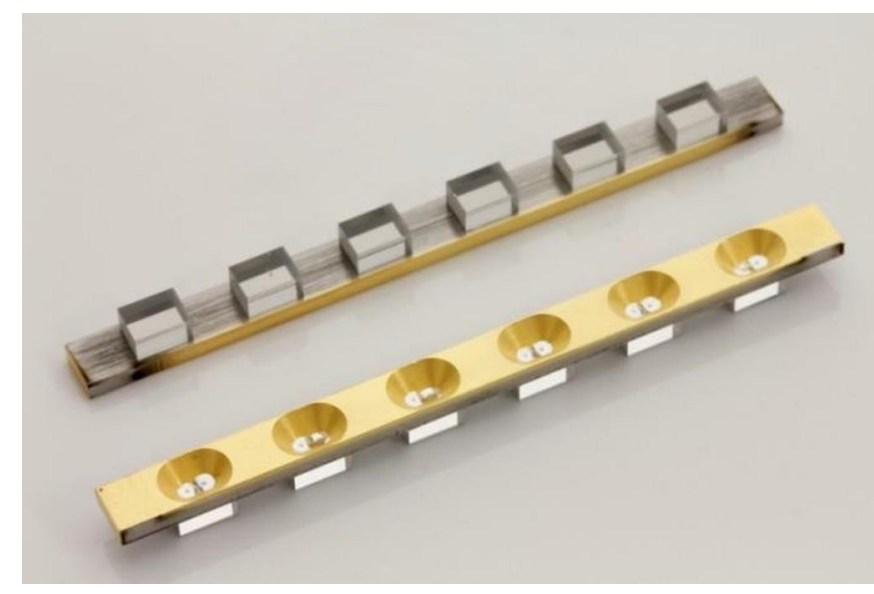

FIG. 1 Soldered sample of $\mathrm{LAK}_{9} \mathrm{G}_{15}$ glass test components and TiAl6 $\mathrm{V}_{4}$ metal mount.

robust solid-state laser for the ExoMars mission [20], and the low stress mounting of lenses [21].

\subsection{Sample preparation}

We investigate Solderjet Bumping as a joining technique for large aperture transmissive optics (diameter approximately $74 \mathrm{~mm}$ made of SQ1 and $50 \mathrm{~mm}$ made of LAK9G15) bonded to thermally matched metallic mounts. Driven by real application specifications these demonstrators are different in geometry. Previous experiments have been conducted to determine suitable parameter sets for the laser-based reflow using the Design-of-Experiments method to evaluate for optimized wetting while avoiding damage to the glass material. The bond strength has been determined and the design of the mount, of flexure hinges, and of the bond area/geometry have been optimized using finite elements analysis [21].

We use the near-eutectic tin-silver-copper (Sn96.5Ag3Cu0.5, SAC305), a common lead-free solder for electronics manufacturing, for this study. The melting range is $217^{\circ} \mathrm{C}$ to $220^{\circ} \mathrm{C}$. The ultimate tensile strength (UTS) of SAC305 ranges from $35 \mathrm{MPa}$ to $70 \mathrm{MPa}$ depending on the microstructure due to different cooling rates and of course depending on the strain-rate of the relevant load case [22]. For the rapid cooling of the solder alloy during Solderjet Bumping, significantly above $300 \mathrm{~K} \mathrm{~s}^{-1}$, and a low strain rate $1 \times 10^{-5} \mathrm{~s}^{-1}$ we assume a conservative worst case UTS of $45 \mathrm{MPa}$ for design of the fixtures and the bond areas. High strain-rate load scenarios, e.g. vibrational and shock loads, will lead to a higher UTS [23].

We reported ultimate shear strengths of bonds by Solderjet Bumping of $55.7 \mathrm{MPa} \pm 6.1 \mathrm{MPa}$ for SQ1 and $54 \mathrm{MPa} \pm 5.4 \mathrm{MPa}$ for LAK9G16 [21], which is in line with above mentioned design estimate. The failure mode for SAC305 solder joints, a bulk solder crack, confirms the suitability of the PVD metallization system and its adhesion strength on the glass material. It suggests the scalability of the absolute bond strength by increasing the bond's cross-section, e.g. larger bond surface and higher amount of solder droplets. The shear strength's variance of $11 \%$ proves the process to be reproducible.

Based on these results we designed basic experiments to 


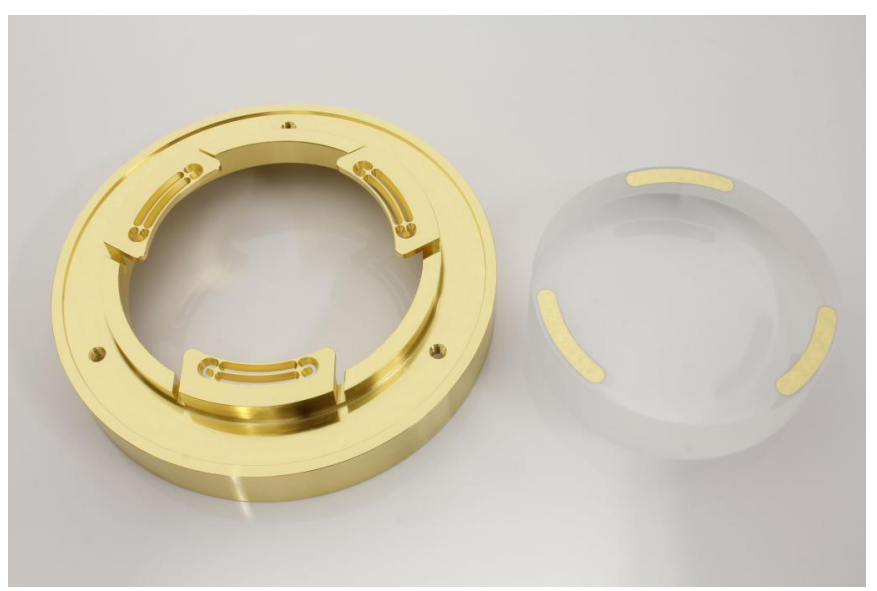

FIG. 2 Soldered lens demonstrator using LAK9G15 mirror blank and TiAl6V 4 metal mount.

determine the OPD due to laser soldering using i) simplified testing vehicles (prismatic glass elements, LAK9G15, $3 \times 2 \times 4 \mathrm{~mm}^{3}$ ) (see Figure 1) and ii) mirror blanks (SQ1 and LAK9G15) with coplanar surfaces according to the physical dimensions and interfaces to lens mounts (see Figure 2). Both kind of sample pairs consist of metal mounts with machined conical holes and the optical elements. Material combinations are selected for a minimized differential thermal expansion: SQ1 is paired with Invar and LAK9G15 with TiAl6V4 with differences in thermal expansion of $-0.7 \times 10^{-6} \mathrm{~K}^{-1}$ and $-1.2 \times 10^{-6} \mathrm{~K}^{-1}$ respectively.

\subsection{Evaluation of optical path difference by photoelasticity measurements}

To determine the stress distribution in the optical material we used a polaricsope (StrainMaticR M4/60.13 zoom, Ilis GmbH, Germany) for the measurement of photoelasticity. The repeatability of OPD measurements with this polariscope is documented to be less than $\pm 0.5 \mathrm{~nm}$. The polariscope's telecentric zoom lens allows selection of a field of view according to the device under test $\left(48 \times 40 \mathrm{~mm}^{2}\right.$ to $12 \times 10 \mathrm{~mm}^{2}$, with respective pixel sizes of $40 \mu \mathrm{m}$ to $10 \mu \mathrm{m}$ ). Test case i) fits completely in the field of view, so the reported values are maxima per sample. Measurements of the three fixtures of the mirror blank demonstrators were done separately and stitched together. For test case ii) the presented results are therefore the mean values of the respective maxima of OPD per sample.

\section{EXPERIMENTAL RESULTS}

Figure 3 shows the evolution of OPD values of LAKG15 test samples along processing and environmental testing. Values given are mean and standard deviation for six samples each. The results document the maximum OPD (full range, peakto-valley (PV)) of the sample within the measurable aperture. From 1) initial values to 2) metallized samples only a minor change is observed that could be contributed to residual thin film layers at the edges of the sample. An expected significant increase of OPD after 3) soldering is measured. Samples are then processed differently: stored at ambient conditions (AS) $\left(22^{\circ} \mathrm{C}\right.$, rel. humidity $\left.40 \%\right)$, at an elevated temperature (ET) of

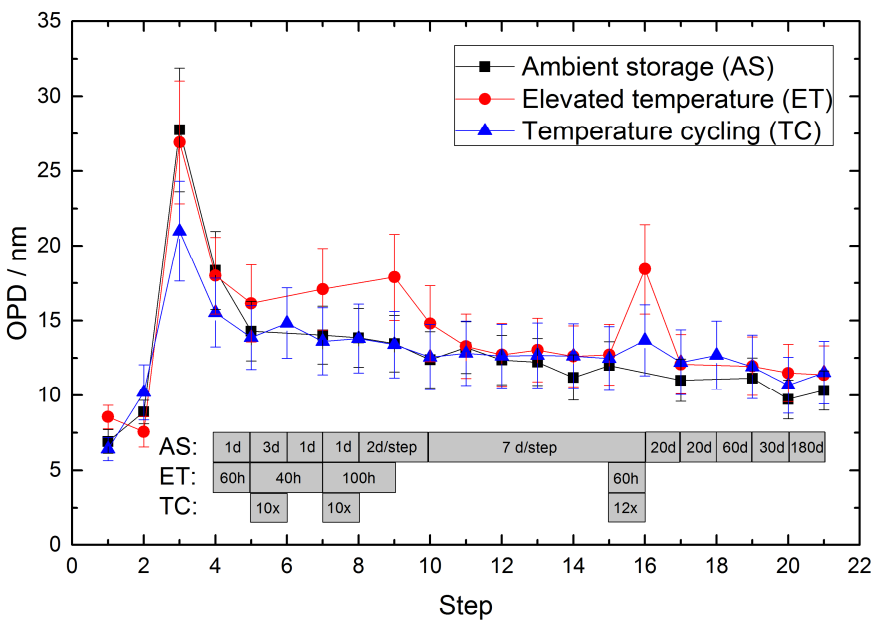

FIG. 3 Optical path difference (full range PV) for $\mathrm{LAK}_{9} \mathrm{G}_{15}$ test samples for all process steps: 1) initial, 2) metallized, 3) soldered, 4) through 21) storage conditions (see text).

$80^{\circ} \mathrm{C}$ with ramp-up/ramp-down of $0.5 \mathrm{~K} \mathrm{~min}^{-1}$, and exposed to temperature cycling (TC) $-40^{\circ} \mathrm{C}$ to $60^{\circ} \mathrm{C}$ at $2 \mathrm{Kmin}^{-1}$. ET and TC samples are stored at ambient conditions between their respective testing cycles.

During ambient storage a decrease in OPD due to relaxation of stresses is observed. Exposure to elevated temperature leads to an increase in OPD, see steps 7), 9), and 16), that recedes back to levels of the unexposed sample. Temperature cycling also slighly rises OPD, see steps 6), 8), and 16), but the values also converge to the respective results of the ambient stored sample. The most significant decrease is detected within the first few days after soldering and measurement intervals have therefore been increased after that initial drop. LAK9G15 shows an increase of OPD (PV) of $3.4 \mathrm{~nm}$ (AS), $2.8 \mathrm{~nm}(\mathrm{ET})$, and $5.1 \mathrm{~nm}$ (TC) after prolonged storage (approximately 400 days). The respective variance of OPD (RMS) after storage is $0.9 \mathrm{~nm}(\mathrm{AS}), 1.6 \mathrm{~nm}(\mathrm{ET})$, and $1.5 \mathrm{~nm}(\mathrm{TC})$.

The results for the mirror blank demonstrators represent four samples per material SQ1 and LAK9G15. We excluded one manufactured SQ1 sample from these results due to its excessively high initial OPD values ( $14 \mathrm{~nm}$ vs. typically less than $5 \mathrm{~nm}$ ). Figure 4 shows arithmetic mean and standard deviation of PV values of OPD for the process steps of 1) initial measurement of the mirror blanks, 2) after metallization, 3) after soldering, 4) through 7) storage, 8) after vibrational test, 9) storage, 10) thermal vacuum test, and 11) to 12) storage. Vibrational tests include resonance search, sinusoidal vibration, and random vibration. The thermal vacuum test covers eight cycles $-40^{\circ} \mathrm{C}$ to $60^{\circ} \mathrm{C}$ at $2 \mathrm{~K} \mathrm{~min}^{-1}$. Between soldering and vibrational test a storage period for the SQ1 samples between 31 and 34 days took place. LAK9G15 samples were kept in storage for 38 to 42 days. The samples were stored at ambient conditions for eleven days (SQ1) and seven days (LAK9G15) between vibrational and thermal evaluation. These differences in duration were necessitated by availability of measurement equipment.

We observe a significant increase and a large deviation between samples after metallization that could be contributed 


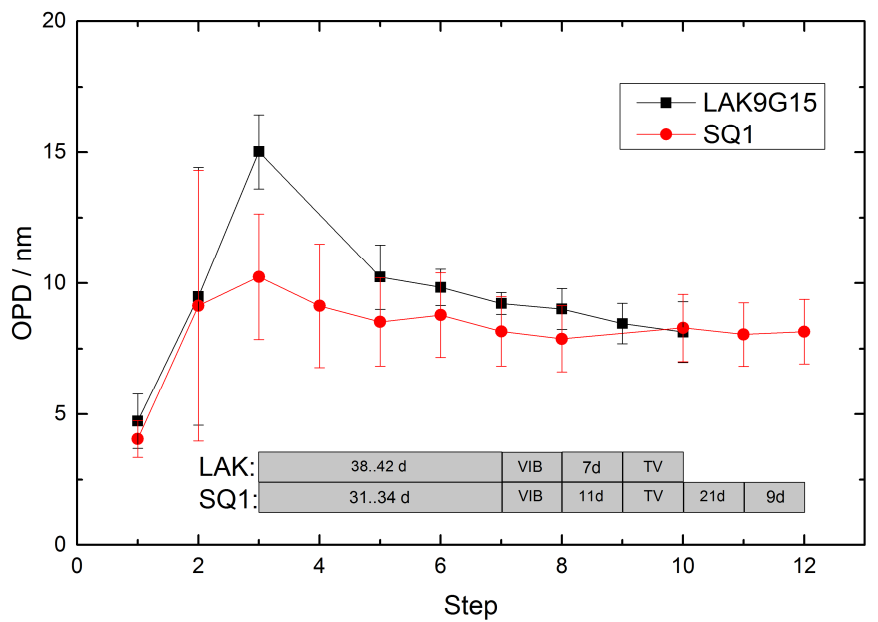

FIC. 4 Optical path difference (full range PV) for $\mathrm{SQ}_{1}$ and $\mathrm{LAK}_{9} \mathrm{G}_{15}$ mirror blank for all process steps: 1) initial, 2) metallized, 3) soldered, 8) vibrational testing (VIB), 10) thermal vacuum cycling (TV), and storage (all else).

to artifacts due to inadvertent residual thin film layers near the bond pads in areas insufficiently covered by vapor shade masking. The induced stresses introduced by soldering significantly increase OPD with a distinctly larger peak for LAK9G15 at $15 \mathrm{~nm}$ vs. SQ1 at $10 \mathrm{~nm}$. A fact that is not reproduced by the type i) samples and might therefore be contributed by a difference in the mechanical fixtures of the two different mirror blanks. Relaxation during storage and vibrational load change these values insignificantly compared to storage under ambient conditions.

For SQ1 we find an increase from initial $4.1 \mathrm{~nm} \pm 0.7 \mathrm{~nm}$ to $8.2 \mathrm{~nm} \pm 1.2 \mathrm{~nm}$ after relaxation. LAK9G15 shows initial values of $4.7 \mathrm{~nm} \pm 1.0 \mathrm{~nm}$ and settles at $8.1 \mathrm{~nm} \pm 1.2 \mathrm{~nm}$. An overall increase of less than $4 \mathrm{~nm}$ (PV) of OPD for soldered lenses dummies is achieved by Solderjet Bumping, with stress distributions localized mainly at the flexures and solder joints. A corresponding change in RMS values of less than $2 \mathrm{~nm}$ is found. Figures 5 and 6 show the false color coded visual representation of the OPD changes for the process and evaluation steps, metallization, soldering, vibrational, and thermal testing. The distinct increase after soldering is clearly visible for both materials. A slight increase of OPD and the respective induced stress is observed after thermal cycling for SQ1. It might be contributed to a plastic deformation of the machined flexures of the mount. Prolonged annealing of the mounts after cutting and milling of these flexures could decrease such influences and should be investigated further. In contrast to SQ1 no increase after thermal cycling was detected for LAK9G15. This indicates that TiAl6V4 is less prone to tensioning after mechanical machining.

\section{CONCLUSIONS}

We investigated the influence of laser-based soldering of optical components on stress induced changes of optical path difference for SQ1 and the radiation resistant glass LAK9G15. Test samples of LAK9G15 show an increase of OPD (PV) of approximately $3 \mathrm{~nm}$ to $5 \mathrm{~nm}$ after prolonged storage. The respective variance (RMS) is less than $1.6 \mathrm{~nm}$. With demonstrator

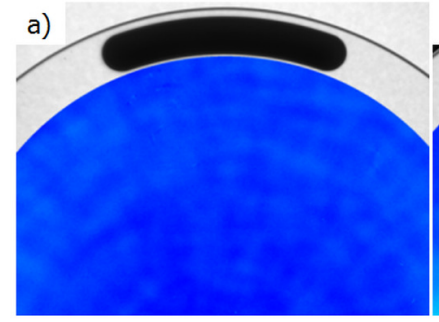

$\mathrm{OPD}_{\max }=4.0 \mathrm{~nm}$

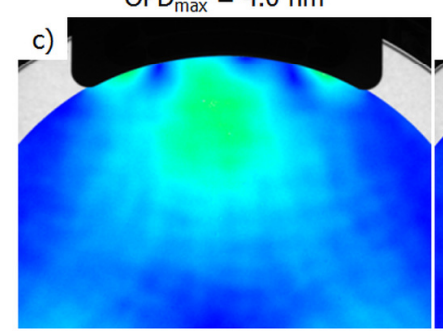

$\mathrm{OPD}_{\max }=7.4 \mathrm{~nm}$

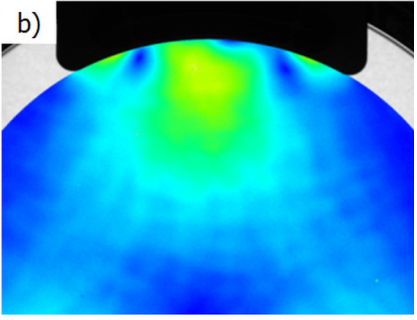

$\mathrm{OPD}_{\max }=10.0 \mathrm{~nm}$

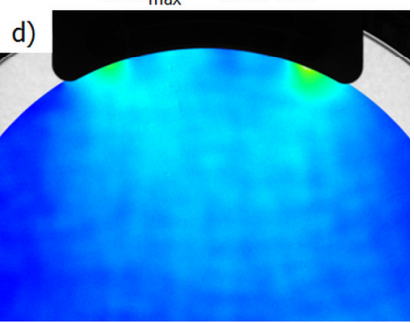

$\mathrm{OPD}_{\max }=9.2 \mathrm{~nm}$
FIG. 5 Visual representation of $O P D$ in $S_{1} / /$ Invar sample for the process steps:

a) metallized, b) soldered, c) after vibrational test, and d) after thermal vacuum.

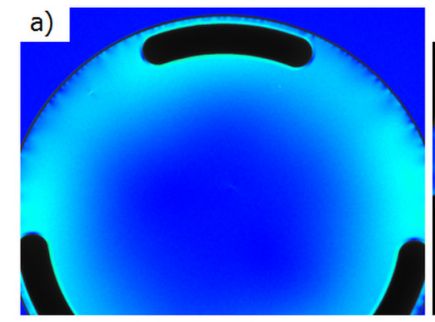

$\mathrm{OPD}_{\max }=6.8 \mathrm{~nm}$

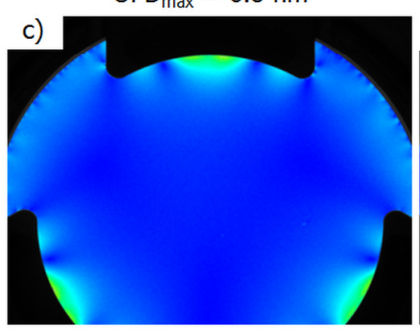

$\mathrm{OPD}_{\max }=8.9 \mathrm{~nm}$

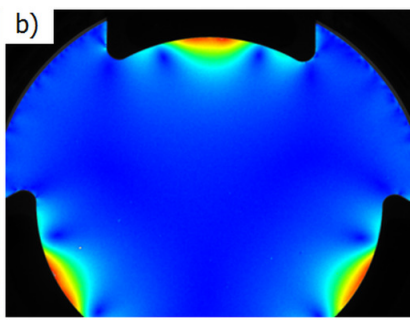

$\mathrm{OPD}_{\max }=15.3 \mathrm{~nm}$

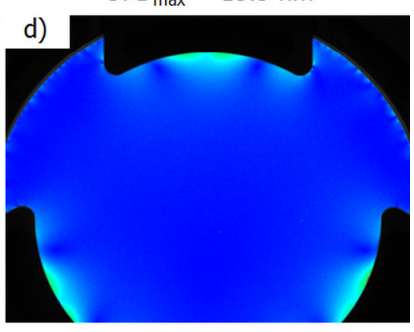

$\mathrm{OPD}_{\max }=7.6 \mathrm{~nm}$
FIG. 6 Visual representation of OPD in LAK9G15/TiAl6V 4 sample for the process steps: a) metallized, b) soldered, c) after vibrational test, and d) after thermal vacuum.

designs close to real application specifications (large aperture lenses, approximately $74 \mathrm{~mm}$, SQ1 and $50 \mathrm{~mm}$, LAK9G15), we report an increase of OPD of less than $4 \mathrm{~nm}$ (PV) after relaxation for both materials. The change in RMS values is less than $2 \mathrm{~nm}$. We found stress distributions localized mainly at the flexures and solder joints.

This overall increase seems acceptable even for components of high quality optical systems. Careful management of the aberration budget may enable the use of Solderjet Bumping for the assembly of such systems. Future work should address the further reduction of stress induced change of OPD and surface deformation to achieve the $1 \mathrm{~nm}$ mark for highend lithography lenses with a tolerable level of aberration of $\lambda / 200$ (RMS) [24].

\section{ACKNOWLEDGEMENTS}

The presented work has been funded by the Federal Ministry for Economic Affairs and Energy (Bundesministerium für 
Wirtschaft und Technologie, BMWi) by resolution of the German Federal Parliament under grant number 50EE1231. The project was administrated by the German Aerospace Center (Deutsches Zentrum für Luft- und Raumfahrt, DLR). The authors would like to thank R. Schmidt, G. Leibeling, S. Müller, M. Scheler, T. Müller, S. Schulze, and S. Gramens for sample manufacturing, preparations, coating, and measurements.

\section{References}

[1] T. Sure, J. Heil, and J. Wesner, "Microscope objective production: on the way from the micrometer scale to the nanometer scale," Proc. SPIE 5180, 283-292 (2004).

[2] J. Heil, T. Bauer, W. Mueller, T. Sure, and J. Wesner, “New tools for high-precision positioning of optical elements in high-NA microscope objectives," Proc. SPIE 5252, 431-442 (2004).

[3] E. Grossman, and I. Gouzman, "Space environment effects on polymers in low earth orbit," Nucl. Instrum. Meth. B 208, 48-57 (2003).

[4] L. Reichmann, 0. Falkenstörfer, U. Krüger, T. Schletterer, M. Bening, and M. Bornschein, "Low-stress glue-free mounting technology for optical elements," Proc. SPIE 7100, 71001B-1-71001B-7 (2008).

[5] H. Holderer, and J. Christ, "UV-resistant jointing technique for lenses and mounts," US Patent 5991101 (1999).

[6] U. Bingel, H. Holderer, and F. Zernike, "Galvanoplastic optical mount," EP Patent 0898189 (2005).

[7] T. Takahashi, T. Okumura, E. Suzuki, T. Kojima, H. Suzuki, T. Tojo, and K. Machida, "Lens-cementing technology used in optical systems of deep-ultraviolet wavelength regions," J. Micro-Nanolith. MEM 6, 043010 (2007).

[8] C. Rothhardt, J. Rothhardt, A. Klenke, T. Peschel, R. Eberhardt, J. Limpert, and A. Tünnermann, "BB0-sapphire sandwich structure for frequency conversion of high power lasers," Opt. Mater. Express $4,1092-1103$ (2014).

[9] S. Sinha, K. E. Urbanek, A. Krzywicki, and R. L. Byer, “Investigation of the suitability of silicate bonding for facet termination in active fiber devices," Opt. Express 15, 13003-13022 (2007).

[10] T. Tamaki, W.Watanabe, and K. Itoh, "Laser micro-welding of transparent materials by a localized heat accumulation effect using a femtosecond fiber laser at 1558 nm," Opt. Express 14, 10460-10468 (2006).

[11] S. Richter, S. Döring, A. Tünnermann, and S. Nolte, "Bonding of glass with femtosecond laser pulses at high repetition rates," Appl. Phys. A-Mater. 103, 257-261 (2011).
[12] K. Egashira, and M. Kobayashi, "Analysis of thermal conditions in $\mathrm{CO}_{2}$ laser splicing of optical fibers," Appl. Optics 16, 2743-2746 (1977).

[13] K. Egashira, and M. Kobayashi, "Optical fiber splicing with a lowpower $\mathrm{CO}_{2}$ laser," Appl. Optics 16, 1636-1638 (1977).

[14] M. Zervas, and C. Codemard, "High power fiber lasers: a review," IEEE J. Quantum Elect. 20, 219-241 (2014).

[15] H. Faidel, B. Gronloh, M. Winzen, E. Liermann, D. Esser, V. Morasch, J. Luttmann, et al., "Passive alignment and soldering technique for optical components," Proc. SPIE 8235, 8235-8240 (2012).

[16] L. Stauffer, A. Würsch, B. Gächter, K. Siercks, I. Verettas, S. Rossopoulos, and R. Clavel, "A surface-mounted device assembly technique for small optics based on laser reflow soldering," Opt. Laser. Eng. 43, 365-372 (2005).

[17] H. Banse, E. Beckert, R. Eberhardt, W. Stöckl, and J. Vogel, "Laser beam soldering- a new assembly technology for micro optical systems," Microsyst. Technol. 11, 186-193 (2005).

[18] E. Beckert, T. Burkhardt, M. Hornaff, A. Kamm, I. Scheidig, C. Stiehl, R. Eberhardt, et al., "Submicron accuracy optimization for laser beam soldering processes," Proc. SPIE 7585, 758505 (2010).

[19] J. Ojeda, E. Beckert, T. Burkhardt, M. Hornaff, and A. Kamm, “Experimental evaluation of the polarization crosstalk when soldering a polarization-maintaining fiber into a v-grooved substrate," IEEE T. Compon. Pack T. 3, 543-548 (2013).

[20] P. Ribes, T. Burkhardt, M. Hornaff, S. Kousar, D. Burkhardt, E. Beckert, M. Gilaberte, et al., "Solderjet bumping technique used to manufacture a compact and robust green solid-state laser," Proc. SPIE 9520, 95200 (2015).

[21] T. Burkhardt, M. Hornaff, A. Kamm, D. Burkhardt, E. Schmidt, E. Beckert, R. Eberhardt, et al., "Low-strain laser-based solder joining of mounted lenses," Proc. SPIE 9574, 95740M (2015).

[22] K. Kim, S. Huh, and K. Suganuma, "Effects of cooling speed on microstructure and tensile properties of Sn-Ag-Cu alloys," Mat. Sci. Eng. A-Struct 333, 106-114 (2002).

[23] P. Lall, S. Shantaram, J. Suhling, and D. Locker, "Effect of high strain-rate on mechanical properties of $\mathrm{SAC}_{105}$ and $\mathrm{SAC}_{305}$ leadfree alloys," in Proceedings to the $62^{\text {nd }}$ Electronic Components and Technology Conference (ECTC), 1312-1326 (IEEE, San Diego, 2012).

[24] B. W. Smith, "Optical projection lithography," in Nanolithography: the art of fabricating nanoelectronic and nanophotonic devices and systems, M. Feldman, ed., 1-41 (Woodhead Publishing, Cambridge, 2014). 